\title{
A SUPPLEMENT TO THE STRONG LAWS FOR WEIGHTED SUMS OF $\varphi$-MIXING RANDOM VARIABLES
}

\author{
Haiwu Huang, Dingcheng Wang, Qunying Wu And Jiangyan Peng
}

Abstract. Some complete convergence theorems for linear statistics that are weighted sums $\sum_{i=1}^{n} a_{n i} X_{i}$ are established, where $\left\{X_{n} ; n \geqslant 1\right\}$ is a sequence of $\varphi$ - mixing random variables and $\left\{a_{n i} ; 1 \leqslant i \leqslant n, n \geqslant 1\right\}$ is an array of constants. As an application, the Marcinkiewicz-Zygmund strong law of large numbers for weighted sums of $\varphi$ - mixing random variables is obtained.

Mathematics subject classification (2010): 60F15.

Keywords and phrases: $\varphi$-mixing random variables, Marcinkiewicz-Zygmund strong laws, weighted sums.

\section{REFERENCES}

[1] Z. D. BAi AND P. E. Cheng, Marcinkiewicz strong laws for linear statistics, Statistics and Probability Letters, 46, (2000), 105-112.

[2] R. L. Dobrushin, The central limit theorem for non-stationary Markov chain, Theory of Probability and Its Applications, 1 (4), (1956), 72-88.

[3] S. A. UTEV, On the central limit theorem for $\varphi$-mixing arrays of random variables, Theory of Probability and Its Applications, 35 (1), (1990), 131-139.

[4] D. C. CHEN, A uniform central limit theorem for nonuniform $\varphi$-mixing random fields, The Annals of Probability, 19 (2), (1991), 636-649.

[5] N. HERRNDORF, The invariance principle for $\varphi$-mixing sequences, Zeitschrift fur Wahrscheinlichkeitstheorie und Verwandte Gebiete, 63 (1), (1983), 97-108.

[6] M. Peligrad, An invariance principle for $\varphi$-mixing sequences, The Annals of Probability, 13 (4), (1985), 1304-1313.

[7] P. K. SEN, A note on weak convergence of empirical processes for sequences of $\varphi$-mixing random variables, Annals of Mathematical Statistics, 42 (6), (1971), 2131-2133.

[8] P. K. SEN, Weak convergence of multidimensional empirical processes for stationary $\varphi$-mixing processes, The Annals of Probability, 2 (1), (1974), 147-154.

[9] J. IOSIFESCU, Limit theorem for $\varphi$-mixing sequences, in Proceedings of the 5th Conference on Probability Theory, 1-6, September, (1977).

[10] M. Peligrad, On Ibragimov-Iosifescu conjecture for $\varphi$-mixing sequences, Stochastic Processes and Their Applications, 35 (2), (1990), 293-308.

[11] Q. M. SHAO, Almost sure invariance principles for mixing sequences of random variables, Stochastic Processes and Their Applications, 48 (2), (1993), 319-334.

[12] S. H. Hu AND X. J. WANG, Large deviations for some dependent sequences, Acta Mathematica Scientia, Series B, 28 (2), (2008), 295-300.

[13] X. J. WANG, et al, Moment inequality for $\varphi$-mixing sequences and its applications, Journal of Inequalities and Applications, (2009), Article ID 379743, 12 pages doi:10.1155/2009/379743.

[14] X. J. WANG, et al, On complete convergence for weighted sums of $\varphi$-mixing random variables, Journal of Inequalities and Applications, (2010), Article ID 372390, 13 pages doi:10.1155/2010/372390.

[15] G. H. CAI, Marcinkiewicz strong laws for linear statistics of $\rho^{*}$-mixing sequences of random variables, Anais da Academia Brasileira de Cişncias, 78 (4), (2006), 615-621. 
[16] C. R. LU AND Z. Y. Lin, Limit theory for mixing dependent sequences, Science Press, Beijing, China, (1997). 